\title{
Application of Evidence-based Nursing in Nursing Quality Management of the Urology Surgical Department
}

\author{
Junna Ma* \\ Department of Surgery, People's Hospital of Changshan County, Quzhou, Zhejiang 324200, China
}

\begin{abstract}
Objective: To explore the application effect of evidence-based nursing in nursing quality management of the Urology Surgical Department, and to provide reference basis for clinical nursing. Method: 80 cases of urology surgical patients from April 2013 to April 2014 treated at our hospital were selected in this study. They were divided into observation group and control group ( 40 cases in each group) according to the nursing method. The control group was given conventional nursing, while the observation group was given evidence-based nursing. Compliance and satisfaction were compared between two groups of patients. Results: After nursing, comparative differences of compliance and satisfaction in patients with two groups are significant $(p<0.05)$. The observation group is better than the control group. Conclusion: The application effect of evidence-based nursing in the urology surgical department's for nursing quality management is remarkable. It improves the patient's satisfaction and treatment adherence, is worthy of extending in clinical.
\end{abstract}

\section{KEYWORDS}

Evidence-based nursing

Urology surgical

Nursing quality

\section{Introduction}

Evidence-based medicine has gradually evolved into evidence-based health care after long time development, and developed into empirical decision-making concepts in nursing and public health at the same time. Evidencebased nursing involves in clinical nursing, nursing staff combined the patient's wishes and clinical experience with research conclusion scientifically and carefully, which make it as a decision basis of the whole process of clinical nursing care. The nursing science which is influenced by evidence-based medicine has subject experienced continuous development and transmute in the past few decades. It is gradually transformed into taking patients as the center

Copyright $\odot 2016$ Junna Ma

doi: $10.18686 /$ jn.v5i2.15

Received: June 30, 2016; Accepted: August 7, 2016; Published online: August 27, 2016

This is an open-access article distributed under the terms of the Creative Commons Attribution Unported License (http://creativecommons.org/ licenses/by-nc/4.0/), which permits unrestricted use, distribution, and reproduction in any medium, provided the original work is properly cited.

${ }^{\star}$ Corresponding author: Department of Surgery, People’s Hospital of Changshan County, Quzhou, Zhejiang 324200, China. E-mail: majunna123y@sina.com of the whole nursing, has achieved a considerable success in practice. This study aimed to promote evidence-based nursing mode further, enrich clinical experience, improve patient's care satisfaction, and improve the relationship between nurses and patients. 80 cases of urology surgical patients who treated at our hospital from April 2013 to April 2014 were selected. 40 patients are given the evidencebased nursing and the nursing effect is remarkable.

\section{Information and method \\ 2.1. General information}

80 cases of urology surgical patients treated at our hospital from April 2013 to April 2014 were selected in this study. They were divided into observation group and control group (40 cases in each group) according to the nursing method. In the observation group, 28 cases are males, 12 cases are females; the age ranged from 23 to 56 years old and average was 39.5 years old. In the control group, 30 cases are males, 10 cases are females; the age ranged from 24 to 56 years and average age was 40 years old. All of the patients do not have serious organs dysfunction such as heart, liver and kidney function. Their consciousness is clear and can make normal communication with medical staff. General information of two groups of patients, such 
as gender, age and condition of illness, do not have significant difference $(p>0.05)$, thus it is comparable.

\subsection{Nursing methods}

The control group was given routine care, including disease observation, monitoring of vital signs, discharge guidance, and etc. Observation group patients were given evidencebased nursing, specific measures are as follows.

\subsubsection{Set up the nursing team}

Experienced nurses were selected to form an evidencebased nursing group. They had to collect and analyze the urology surgical department's clinical situation and common problems, evaluate the most serious problems the urology patients have, including psychological pressure and complications.

\subsubsection{Evidence-based support}

The nurses had to consult relevant statistical data actively, develop scientific and reasonable nursing plan aiming at the problem of urology patients. Theoretical basis for improving the quality of nursing was provided.

\subsubsection{Hospitalizing education}

When patients admitted to hospital, the urology disease's prevention knowledge was explained to patients and their families in details by appointed nurse, to make a preliminary understanding of damage caused by urology. Nurses explained the treatment principle, advantages and safety related knowledge of urological diseases in details to them after warded. This helps patients to eliminate tension, and actively cooperate with treatment and related inspection.

\subsubsection{Application of evidence-based}

Different health education method was chosen which mainly including psychological nursing, diet nursing and health education, according to patients' individual differences. Plenary communication and exchanges with patients and their families were conducted timely to understand the illness development situation and treatment measures and to promote nursing quality. As most of the surgical patients need surgery, relevant personnel should be prior to surgery for patients with sufficient guidance. This can relieve their inner anxiety and make them actively cooperated with treatment. Nursing staff was combined with the practical situation of patients to set high standards of nursing needs, and improve nursing model and also patient's care satisfaction.

\subsubsection{Urine tube indwelling care}

In nursing, $\mathrm{pH}$ value of 6.8 was set as the cut-off point. If the patient's urine was $\mathrm{pH}>6.8$, urine tube was changed once every two weeks. If urine was $\mathrm{pH}<6.8$, urine tube was changed once every 3 to 4 weeks.

\subsection{Observation index}

The compliance and satisfaction of the two groups were compared. Compliance is including Good: patients can accept treatment on time in accordance with the doctor's advice; General: patients need the supervision of nurses or family members; and Invalid: patients refuse treatment. Two groups were compared with good rates. Satisfaction is divided into bad, good and superior through the self-made form of patient's satisfaction. The content is mainly related to nurses' service attitude, health education, life nursing, nursing technical operation, and psychological care measures. Results were analyzed after patients discharge.

\subsection{Statistical treatment}

SPSS15.0 statistical software was used for data analysis. The measurement data using \pm standard deviation, using $t$ test, counting data with $\chi^{2}$ test, with $p<0.05$ for the difference is significant.

\section{Results}

\subsection{Patient's compliance}

After nursing, the observation group of patients with good compliance rate was $85.00 \%$ and the control group in good rate was $42.50 \%$. Difference between two groups of patients was significant $(p<0.05)$, observation group is better than the control group. Concrete results were shown in Table 1.

Table 1. Comparison of two groups of patient's compliance (\%).

\begin{tabular}{ccccc}
\hline Group & Cases & Good & General & Invalid \\
\hline Observation group & 40 & $35(87.50)$ & $4(10.00)$ & $1(2.50)$ \\
Control group & 40 & $27(67.50)$ & $6(15.00)$ & $7(17.50)$ \\
\hline
\end{tabular}

\subsection{Comparison of satisfaction}

After nursing, patient satisfaction good rate of observation group is $97.50 \%$ and control group is $87.50 \%$. Difference between two groups was significant $(p<0.05)$. The observation group is better than the control group. Concrete results were shown in Table 2.

Table 2. Comparison of two group's satisfaction situation (\%).

\begin{tabular}{cccccc}
\hline Group & Case & Bad & Good & Superior & $\begin{array}{c}\text { Good } \\
\text { rate(\%) }\end{array}$ \\
\hline $\begin{array}{c}\text { Observation } \\
\text { group }\end{array}$ & 40 & $1(2.50)$ & $5(12.50)$ & $3(85.00)$ & 97.50 \\
$\begin{array}{c}\text { Control } \\
\text { group }\end{array}$ & 40 & $5(12.50)$ & $8(20.00)$ & $2(67.50)$ & 87.50 \\
\hline
\end{tabular}

\section{Discussions}

Demands for hospital care are increased with the progress of time, per capita material life level has improved. Clinical practice has certain nursing experience and the theoretical basis after a long-term development and perfection. It has achieved a considerable success in the practical appli- 
cation. The formation of nursing mode gives the comprehensive and effective nursing intervention mainly from the divergent perspective on the patients. It also prevents the occurrence of complications, eases the pain of the patients and improves patients' body function recovery and quality of life. Urology is a clinical common disease and frequently encountered disease, although after effective treatment. However, due to the nursing work does not reach the designated position, the deterioration rate was caused by the urinary system infection which has been showing a rising trend and increasing the burden of patients. Clinical studies have found that effective nursing measures have a direct relationship with the prognosis of patients with urology diseases [1]. Patients pay more and more attention to quality of nursing with people's requirement of improving the medical services. Therefore, evidence-based nursing in nursing work becomes the patient's needs.

In clinical practice, the primary basis of evidence-based nursing and nursing work need high comprehensive quality, analysis ability and the strain's ability to handle events. The nursing work brings in more frontier nursing concept, improves and enhances the level of comprehensive nursing care, and provides more professional nursing. Evidencebased nursing should be applied to the urology disease patients as it can be seen as the empirical foundation. The steps can be divided into questions raising, looking for evidence and nursing intervention. The practice of evidencebased nursing intervention mode is based on nurses' clinical experience and professional quality, which influenced by evidence-based medicine and the new nursing concept. Its core idea is to provide quality services according to existing evidence for patients, ensure that nursing staff can make more accurate decisions. Achievements of evidencebased nursing are based on the related fields of scientific research. Compared with common theory, evidence-based nursing is focused on reliable evidence, followed by the scientific basis, based on the nursing staff's practical skills, to select the correct nursing service, realize the organic combination and improve satisfaction. The evidence-based nursing model is widely praised by people in the related field since its application and relieved the relationship between nurses and patients to some extent. Evidence-based nursing paid attention to follow the scientific and reasonable basis, combining with the experience of clinical nursing staff and professional quality. Besides, it is combined with the patient's own condition, with safe, effective and economic benefit for the principle, to carry out the work target and encourage patients to get more high quality nursing service.

In conventional nursing, catheter replacement time is once a week, but the clinical studies found that the catheter changing too frequently is exacerbating the patient's pain, especially for some patients with hyperplasia of prostate, the pain is unbearable to them [2]. It also raises the risk of urinary tract infections, aggravates the burden of the patients, and causes dissatisfaction to the patients and their families with nurse. Therefore, many scholars suggested extending of the catheter time. Some scholars also found that microbial reproduction and urine sediment is a key factor of catheter jam, while microbial propagation and urine sediment is closely related with the patient's urine $\mathrm{pH}$ value [3]. So, $\mathrm{pH} 6.8$ was set as the cut-off point in our hospital to provide targeted nursing. Evidencebased nursing compared with the traditional nursing mode showed that the traditional nursing mode is too rigid, cannot carry out pertinent nursing work, risk of infection is larger and lead to tension between nurses and patients. Evidence-based nursing mode paid more attention to the selection of specific clinical nursing decisions, combined with their own knowledge and experience to guide clinical work.

In relevant literature, patients with urological disease especially in older patients, due to pelvic floor muscles contracted, makes the bladder function impairment and reduces bladder capacity, which greatly increases the instability of the bladder and sensibility. Thus, they are prone to have stress and urinary incontinence. In clinical, microwave physiotherapy, electrical stimulation therapy, and the training of strengthen the bladder and pelvic floor muscles can effectively improve the phenomenon of stress urinary incontinence. Therefore, nurses can guide patients for anus training and urethra contract training.

To summarize, evidence-based nursing mode helps to choose reasonable nursing care decisions to develop a plan of care. On the other hand, it will help to improve patient's satisfaction and the relationship between nurses and patients. From our results, implementation of evidence-based nursing, treatment adherence and patient's satisfaction are improved which are worthy of clinical popularization and application.

\section{References}

1. Gao S, Zhou N, Liu B, et al. Application of evidenceBased Nursing in the Nursing Quality Management of Department of Urology Surgical. China Medical Review. 2007;2007(17):39-41.

2. Zhang A, Zhang $X$. The Effect Observation of EvidenceBased Nursing in the Nursing Application of Department of Uropoiesis Surgical. Journal of Shanxi Medical Journal. 2008; (5):468-470.

3. Zheng $\mathrm{H}$, Zhu GQ, Lin W. Effect of Evidence-Based Nursing on Nursing Quality of Department of Uropoiesis Surgical. Journal of Nursing. 2011;15(5):306-308. 